\title{
Location model of CMS for vehicle navigation in highway network
}

\author{
Dongbin $\mathrm{Xu}^{1,2 \mathrm{a}}$ * \\ ${ }^{1}$ Research Institute of Highway Ministry of Transport, China \\ ${ }^{2} \mathrm{RIOH}$ Transport Consultants LTD., No.8, Xitucheng Road, Beijing, China \\ adb.xu@rioh.cn
}

Keywords: CMS, Location model, highway network

Abstract. Based on benefits evaluation, a location model of CMS is provided in the paper. According to the character of the highway network, some parameters are simplified to calculate traffic flow and attenuation coefficient in the model. The results verify the rationality and practicality of the method.

\section{Introduction}

Changeable message sign (CMS) is one of the important means for releasing information of traffic guidance system. The CMS is also referred to as Variable Message Sign(VMS). In China, the highway is constructed by section because of different investors. In order to meet the requirements of administration, most CMS are usually set up with construction. But from the point of view of the inter-road section and the whole network, the CMS may be set up unreasonably or insufficiently. It will seriously hamper the monitoring and service of the highway network, resulting in a huge waste of investment. Therefore how to address the locations of CMS on condition of highway network to maximize their effectiveness and minimize their numbers becomes very important.

\section{Review of CMS location model}

Abbas and McCoy [1] study the problem of optimizing the locations of CMS for road network firstly. The research goal is to minimum delays, which are produced by vehicles transferred to alternative roads after the drivers responding to the traffic information of CMS, and to estimate delay time by deterministic queuing model on condition with or without CMS. In 2001, Chiu YC proposed a two-level random integer programming model to solve the CMS location problem [2]. The method needs more computational resource and is also unpractical to consider all sections with the same accident rate. In 2005, Liping Fu et al [3] proposed a determined queuing delay model by estimating the benefit of the given CMS, which doesn't consider benefits of CMS under special conditions. Jingyang Zhao et al [4] proposed an optimum CMS location model based on the theory of typical traffic lines to study how to maximize the benefits of variable message signs in a certain area. Fujian $\mathrm{Ni}$ and Zhichao Liu [5] proposed a programming integer model based on effect index. The model defines the effect as the product of traffic and its effect index. It emphasizes on the induction influence and utilizes traffic information of network. The paper [6] proposes a revised mathematical programming model to establish a detailed evaluation index system with theoretical analysis and identification of VMS influence. Liang Li [7] proposes a method for CMS layout using cross-classification and comprehensive evaluation and establishes an optical mathematical model according to the effect of CMS.

Incident delay model based on deterministic queuing model doesn't explain the effect of congestion, traffic saturation, traffic accident rate and transfer traffic volume. Moreover, the applicability of the model in the urban road network weren't be researched $[1,3]$. Theoretically, the location model based on benefits evaluation [6,7] is very suitable for CMS distribution with funding constraints. However, the benefit is difficult to quantify, especially difficult for quantifying the social benefits, and some parameters can't rationally be selected, which limits its using. From theoretical analysis, the mathematical programming model based on impact exponent is very reasonable. Moreover, the structure of the mode is very simple. But the defect of the model is impact exponent only considering 
degree of vehicles affected by one CMS in one road. Therefore, the model has full consideration for the traffic path, which makes it difficult to use. The models mentioned above are suitable for road network in the urban. The paper proposes an improved model for setting up CMS in highway network, which considers characteristic of all the models and actual requirements fully.

\section{CMS location model base on navigation}

Definition. Different roads have different features such as lengths, widths and road conditions. The probability of accident and congestion varided also. So each road has own traffic information. For simplicity, only the most important information is considered. The traffic information of road $j$ is defined as

$$
f_{j}=\left(u_{j}+v_{j}+w_{j}\right) / 3
$$

where $f_{j}$ indicates the traffic information. $u_{j}$ denotes the probability of accident of road $j$, which is computed by the ratio of accident number in the road and that of the road network. $v_{j}$ indicates the probability of traffic jam, which is computed by $V / C$ (the ratio of maximum service traffic volume and the basic capacity). $w_{j}$ is the ratio of the number of disastrous weather days in a year. In Eq.1, the values of all parameters meet [0,1]. When a CMS is placed in different sections, it will show the different effective information. Then the benefit is also different. In order to evaluate the benefits of CMS placed in road $i$ to display information of road $j$, the benefit is defined as $V_{i j}=\alpha_{i} f_{j}$, where $a_{i}$ is annual average daily traffic of road $i$ with annual average daily traffic of the whole network, $a_{i}=q_{i} / \sum q_{i}, q_{i}$ is annual average daily traffic (AADT) of road $i, l$ is the number of all road sections.

To maximize the benefits of CMS, it should be placed in reasonable location where more information showed by CMS as possible and can be seen by more travelers. The effectiveness of CMS is varied according to distance of CMS and information source, which is represented by the attenuation coefficient $e_{i j}$. The distance is larger, the effectiveness is less. Otherwise, the effectiveness is larger. The benefit is redefined as

$$
V_{i j}=e_{i j} \alpha_{i} f_{j} \text {. }
$$

CMS location model. According to the value of CMS, the location of CMS is decided by the information displayed and the number of travelers. So the mathematical programming model is defined as

$$
\max F=\sum_{i}^{l} \sum_{j=R_{i}} X_{i} V_{i j}, \text { s.t. } X_{i}=0,1
$$

where $F$ is the effectiveness of CMS and $l$ is the total number of road sections. $R_{i}$ is the distance set between road section $j$ and road section $i$, which is less than predefine value. If $X_{i}$ equates 1 , the CMS was set up in road $i$. Otherwise none CMS is to be set up. According to the study of road traffic signs information overload threshold, three messages displayed in one sign is recommended. So the $j$ is not more than 3 .

The mathematical programming model includes two aspects. The first one is the number of CMS. It is unpractical that more CMS are set up because of cost too much. Moreover the greater density of CMS will affect safe driving. The second one is whether the CMS will set up or not on road section $i$. Considering economy and safety, the number of CMS is always less than the total number of optional road sections. 


\section{Simplification and solution of model}

Simplification of model. In china, toll stations are set up along the highway on ramps. Through the ramps, vehicles can enter into or exit from the highway. Nowadays, vehicle detectors are used to measure traffic flow on the highway, but the whole road network is not covered with it yet. So it is difficult to obtain the traffic data of any cross-section. In the highway network, the system interchange provides continuous and quick traffic transfers. Considering the function of CMS, the exit-entering volume of observing spot should be nearby the system interchange. In theory, the smaller the distance between the observing spot and the system interchange is, the higher the accuracy of the exit-entering volume is. While in the application of induction service, the users should be informed about traffic information in advance so that they can re-select substituted path. So the observing spot should be setup on 3 or 4 ramps ahead the system interchange. The Fig. 1 shows the relative position of observing spot and the system interchange. So the AADT is obtained at the observing spot.

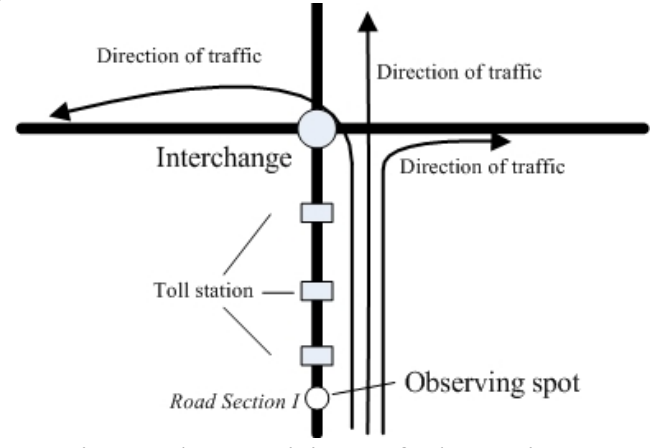

Fig. 1 The position of observing spot

Table 1 The conversion coefficient

\begin{tabular}{c|l|c}
\hline Vehicle classification & \multicolumn{1}{|c}{ Main vehicle's type } & conversion coefficient \\
\hline The first class & $\leqslant 7$ seat(bus) or $\leq 2$ ton(truck) & 1 \\
\hline The second class & $8-19$ seat(bus) or $2-5$ ton(truck) & 1 \\
\hline The third class & $20-39$ seat(bus) or $5-10$ ton(truck) & 3 \\
\hline The fourth class & $\geqslant 40$ seat(bus) or $10-15$ ton(truck) & 3 \\
\hline The fifth class & $\geqslant 15$ ton or container smaller than 40 inches & \\
\hline
\end{tabular}

Actually, the traffic flow is counted in different classification types by traffic management department and can not to be applied to the model directly. Commonly, it should be converted to per car unit. Table 1 shows the coefficient between classification types and per car unit.

As shown in Eq. 2, the attenuation coefficient only depends on the distance between road section $j$ and road section $i$. The result is more reasonable owing to introducing the factor. For travelers, they focus on the adjacent road section because the density of highway network is less than that of urban network. For simplification, we only consider the effect of adjacent road sections. It makes the problem more reasonable and easy computing.

Solution of the model. In traditional mathematical modeling algorithm, Genetic algorithms are uniquely suitable to solve the location problem. But the method and processes are more complex. Considering the convenience and operability, the model can be solved by dynamic programming method. On condition of the fixed total number of road sections, location of CMS model is also be seen as a resource allocation problem. Eq. 3 shows the CMS will be set up on the road sections, which means the product of traffic flow and information is larger. 


\section{Experimental results}

Highway network. In order to test our algorithm, the real highway network is used in the experiment. As shown in the Fig. 2, road sections are showing by full lines and dotted liens because they are owned by different administration departments. Only the segments in full lines are researched in the experiment. For clarity, labels with number are used to indicate the interchanges and virtual interchanges. The lable 5 is one interchange of road sections. Virtual interchanges mean the terminal of road sections or boundaries between different administration departments.

The traffic information. Some data used in the experiment can not be obtained because some certain reasons and some data are unused in the experiment. According to Eq.1, the traffic information $f_{j}$ is shown in the Table 2. Annual average daily traffic of road is shown in Table 3.

According to the Eq. 3, dynamic programming method is used to solve the problem. If the total number of the CMS is 40, the layout of CMS is showed in the following table. In Table 4, Up means the direction meeting with that of the road segment. For example, direction from interchange 4 to interchange 5 of road segment 4-5 has none CMS, while the negative direction should be set up one CMS. The results are consistent with the needs of actual requirements.

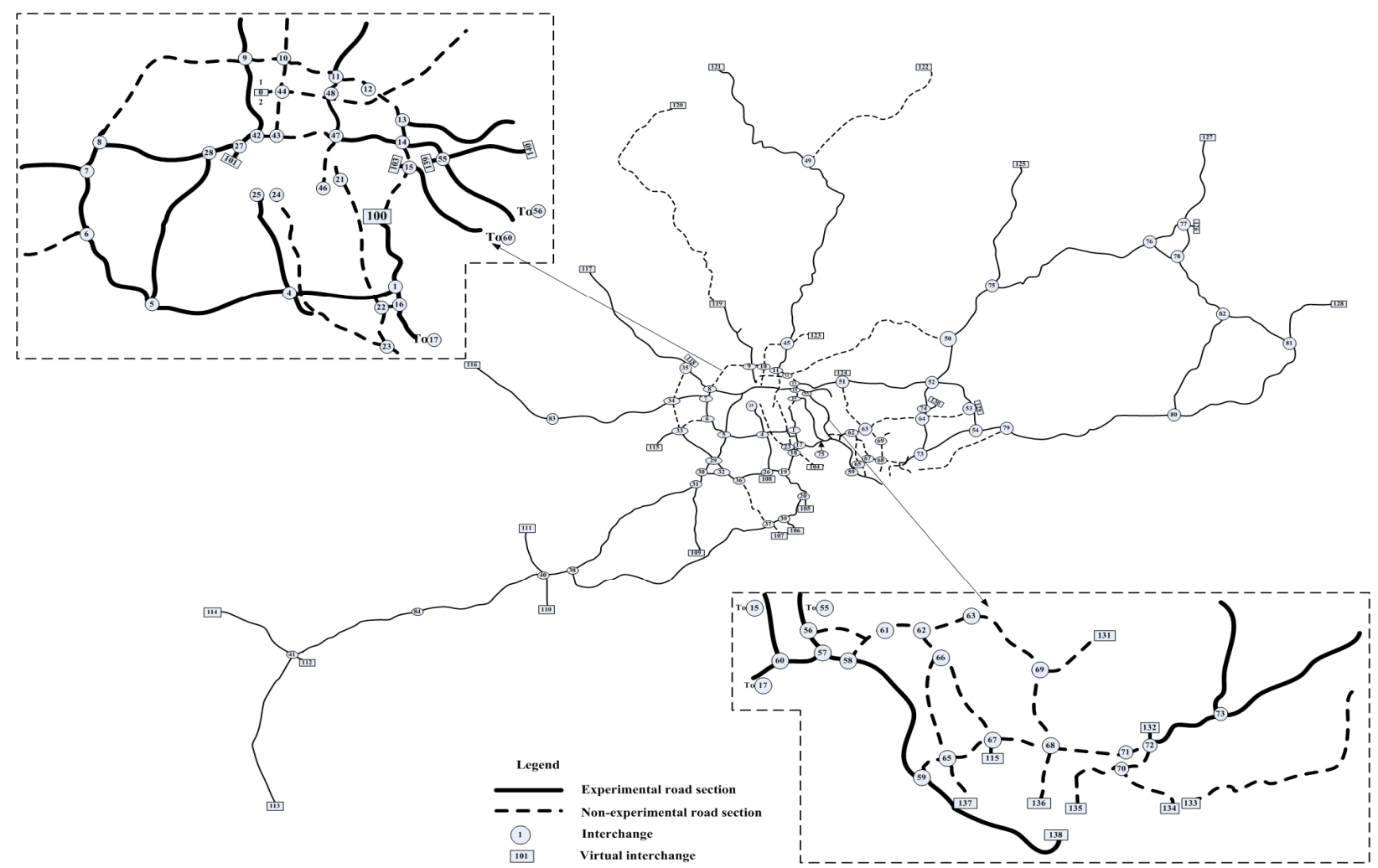

Fig.2 Highway network in experiment 
Table 2 The traffic information

\begin{tabular}{|c|c|c|c|c|}
\hline Road segments & $f_{j}[\%]$ Road segments & $f_{j}[\%]$ Road segments & Road segments & $f_{j}[\%]$ \\
\hline $1-4-5$ & $0.0630-32-36$ & $0.4660-17$ & $10.180-81-128$ & 0.22 \\
\hline $5-6-7-8$ & $\begin{array}{l}0.28 \\
\end{array}$ & \begin{tabular}{|l|l|}
0.99 & $64-73$ \\
\end{tabular} & $0.7180-82$ & 0.03 \\
\hline $7-34$ & $1.9834-83$ & \begin{tabular}{l|l|}
$0.5673-54-79$ \\
\end{tabular} & $4.2483-116$ & 1.43 \\
\hline $8-35-117$ & \begin{tabular}{l|l|}
$0.1236-26-19$ \\
\end{tabular} & \begin{tabular}{l|l|}
1.18 & $73-64-74$ \\
\end{tabular} & $0.46100-1-16-17$ & 0.28 \\
\hline $11-45-49$ & \begin{tabular}{ll|l|}
7.35 & $40-84$ \\
\end{tabular} & $\begin{array}{l}0.65 \\
05-76 \\
\end{array}$ & $0.37110-40-41$ & 0.03 \\
\hline $15-50-52-74-64$ & $5.6141-84$ & $0.6075-125$ & $2.48112-41-114$ & 0.03 \\
\hline $\begin{array}{l}17-18-19-20-39- \\
37\end{array}$ & $2.4841-113$ & $0.1976-77$ & $0.2281-82$ & 0.13 \\
\hline $25-4-26$ & $0.0342-9$ & $0.9376-78$ & $0.0377-127$ & 0.13 \\
\hline $28-5-29-30$ & $6.0742-27-28$ & $2.2977-78-82$ & 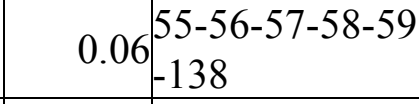 & 35.38 \\
\hline $28-8$ & $1.8049-121$ & $9.60 \mid 79-80-81$ & 0.43 & \\
\hline Subtotal & $25.78-$ & $\begin{array}{l}17.45 \\
\end{array}$ & 19.1 & 37.66 \\
\hline
\end{tabular}

Table 3 Annual average daily traffic

\begin{tabular}{l|c|l|c|l|l|l|l|l|l|l|l}
\hline $\begin{array}{c}\text { Road } \\
\text { segments }\end{array}$ & $\alpha_{i}[\%]$ & $\begin{array}{c}\text { Road } \\
\text { Segments }\end{array}$ & $\alpha_{i}[\%]$ & $\begin{array}{c}\text { Road } \\
\text { segments }\end{array}$ & $\alpha_{i}[\%]$ & $\begin{array}{c}\text { Road } \\
\text { Segments }\end{array}$ & $\alpha_{i}[\%]$ & $\begin{array}{c}\text { Road } \\
\text { Segments }\end{array}$ & $\alpha_{i}[\%]$ & $\begin{array}{c}\text { Road } \\
\text { segments }\end{array}$ & $\alpha_{i}[\%]$ \\
\hline $1-4$ & 0.13 & $28-5$ & 1.09 & $39-106$ & 0.25 & $45-49$ & 0.81 & $80-81$ & 0.31 & $73-64$ & 0.66 \\
\hline $4-5$ & 0.18 & $5-29$ & 0.75 & $38-40$ & 0.47 & $49-121$ & 0.49 & $81-128$ & 0.29 & $64-74$ & 0.59 \\
\hline $5-6$ & 0.24 & $29-30$ & 0.63 & $40-110$ & 0.05 & $49-122$ & 0.16 & $82-81$ & 0.14 & $74-52$ & 0.39 \\
\hline $6-7$ & 0.47 & $30-31$ & 0.84 & $40-111$ & 0.05 & $13-51$ & 0.76 & $82-80$ & 0.17 & $52-50$ & 0.53 \\
\hline $7-8$ & 0.64 & $30-32$ & 0.44 & $40-41$ & 0.35 & $51-52$ & 0.74 & $27-28$ & 1.32 & $50-75$ & 0.49 \\
\hline $100-1$ & 0.16 & $32-29$ & 0.16 & $41-113$ & 0.11 & $52-53$ & 0.44 & $39-20$ & 0.11 & $75-125$ & 0.36 \\
\hline $1-16$ & 0.30 & $29-33$ & 0.13 & $41-114$ & 0.18 & $53-54$ & 0.46 & $11-45$ & 0.85 & $75-76$ & 0.15 \\
\hline $16-17$ & 0.31 & $32-36$ & 0.50 & $41-112$ & 0.21 & $47-14$ & 3.21 & $72-73$ & 0.40 & $76-77$ & 0.08 \\
\hline $17-18$ & 1.32 & $36-26$ & 0.75 & $33-115$ & 0.07 & $14-55$ & 4.06 & $79-80$ & 0.58 & $77-127$ & 0.11 \\
\hline $19-19$ & 1.08 & $26-19$ & 1.02 & $7-34$ & 0.27 & $55-56$ & 15.05 & $59-138$ & 3.58 & $76-78$ & 0.03 \\
\hline $20-105$ & 0.74 & $31-109$ & 0.09 & $34-116$ & 0.40 & $56-57$ & 15.0 & $54-79$ & 0.66 & $77-78$ & 0.16 \\
\hline $25-4$ & 0.25 & $31-38$ & 0.58 & $28-8$ & 0.64 & $57-58$ & 15.55 & $35-117$ & 0.15 & $78-82$ & 0.17 \\
\hline $4-26$ & 0.58 & $38-37$ & 0.11 & $8-35$ & 0.53 & $58-59$ & 12.7 & $37-39$ & 0.25 & $73-54$ & 0.37 \\
\hline
\end{tabular}

Table 4 CMS location of the network

\begin{tabular}{c|c|c|l|c|c|c|c|c|c|c|c}
\hline $\begin{array}{l}\text { Road } \\
\text { segments }\end{array}$ & Up & Down & $\begin{array}{l}\text { Road } \\
\text { segments }\end{array}$ & Up & Down & $\begin{array}{l}\text { Road } \\
\text { segments }\end{array}$ & Up & Down & $\begin{array}{l}\text { Road } \\
\text { segments }\end{array}$ & Up & Down \\
\hline $4-5$ & 0 & 1 & $28-5$ & 1 & 1 & $57-58$ & 1 & 1 & $28-8$ & 1 & 0 \\
\hline $5-6$ & 1 & 0 & $5-29$ & 1 & 1 & $58-59$ & 1 & 1 & $11-45$ & 0 & 1 \\
\hline $16-17$ & 0 & 1 & $29-30$ & 1 & 1 & $59-138$ & 1 & 0 & $45-49$ & 1 & 1 \\
\hline $17-18$ & 1 & 1 & $30-31$ & 1 & 0 & $73-64$ & 1 & 0 & $49-121$ & 1 & 0 \\
\hline $18-19$ & 1 & 1 & $30-32$ & 1 & 0 & $54-79$ & 1 & 1 & $53-54$ & 0 & 1 \\
\hline $19-20$ & 1 & 0 & $32-29$ & 0 & 1 & $79-80$ & 1 & 0 & $55-56$ & 1 & 1 \\
\hline $27-28$ & 1 & 1 & $26-19$ & 0 & 1 & $31-38$ & 1 & 0 & $56-57$ & 1 & 1 \\
\hline Subtotal & 5 & 5 & - & 5 & 5 & - & 7 & 3 & - & 5 & 5 \\
\hline
\end{tabular}




\section{References}

[1] Abbas M, McCoy P, Optimizing variable message sign locations on freeways using genetic algorithms, Transportation Research Board 78th Annual meeting. Transportation Research Board, 1999.

[2] Chiu Yi-Chang, Huynh Nhan, Mahmassani Hani S, Determining Optimal locations for VMS's under stochastic incident scenarios, Transportation Research Board 80th Annual meeting, Transportation Research Board, 2001.

[3]Liping Fu, Shuo Li, Jeff Henderson, Journal of transportation systems engineering and information technology. 5(2005)101-120.

[4] Li Yue. Research on key problems of urban traffic guidance system and its variable message signs, Beijing Jiao Tong University, Beijing, 2006, In Chinese.

[5] Fujian Ni, Zhichao Liu, Information and Control, 32(2003)395-398, In Chinese.

[6] Xiaoqiang Li, Research on the optimal variable message signs(VMS) location problem, Beijing Jiao Tong University, Beijing, 2008, In Chinese.

[7] Liang Li. Study on variable message signs location method based on network, Beijing Jiao Tong University, Beijing, 2012, In Chinese. 